\title{
The Cosmic Microwave Background: An Experimentalists's Guide to CMB Measurements and Prospects for the Future
}

\section{Laura B. Newburgh*}

Dunlap Institute, University of Toronto

E-mail: newburgh@dunlap.utoronto.ca

\begin{abstract}
Measurements of the Cosmic Microwave Background (CMB) have provided an increasingly precise characterization of the Universe and its constituents: matter, radiation, dark matter, and dark energy. These measurements, in conjunction with other cosmological probes including observations of supernova, galaxy surveys, and additional local measurements of the Hubble constant, have contributed crucial evidence for the cosmological model known as $\Lambda$ CDM. Upcoming generations of CMB instruments are poised to constrain additional models that describe the properties of the Universe when it was $<10^{-34}$ seconds old and improve our understanding of large scale structure formation. In this proceedings I will describe the CMB and discuss the status of current measurements and prospects for the future.
\end{abstract}

Frank N. Bash Symposium 2013: New Horizons in Astronomy (BASH 2013)

October 6-8, 2013

Austin, Texas

${ }^{*}$ Speaker. 


\section{Introduction}

Evidence from a variety of cosmological probes and astronomical measurements support that the Universe today is composed of $\sim 4 \%$ standard model particles, $\sim 25 \%$ dark matter, and $\sim 70 \%$ dark energy [18]. This cosmological model is known as $\Lambda \mathrm{CDM}$ and consists of 6 total standard parameters. Measurements from the CMB have been critical both in verifying this model and in providing the most precise constraints on its parameters. Next generation measurements of the $\mathrm{CMB}$ go beyond $\Lambda \mathrm{CDM}$ to probe the very earliest Universe, potentially inform particle physics models, and help enrich our understanding of the formation of the cosmic web. In Section 2 I will give a brief description of the anisotropies in $\mathrm{CMB}$ and what we can learn from them, in Section 3 I will describe the current status of CMB measurements and cosmological constraints, and in Section 4 I will describe the motivations for future CMB measurements. I will not be able to do justice to this large and complicated topic, but the textbook [5] is a particularly good reference for this material.

\section{The Cosmic Microwave Background Anisotropies}

\subsection{The Formation of the CMB}

The CMB was first measured to be uniform background radiation with constant intensity [17]. Its uniformity was evidence that the Universe had been much smaller in the past, but also signaled that something extreme must have occurred in the very early Universe. For the Universe to be so uniform requires that it rapidly expanded, by nearly 30 orders of magnitude in a fraction of a second, to ensure that regions that began in causal contact prior to this expansion did not have an opportunity to re-thermalize. The most popular and simplest explanation is provided by the as-yet unproven theory of inflation (for more information, the textbook [12] has a thorough treatment). The frequency spectrum of the CMB was also measured [13] and found to adhere strictly to a black body spectrum, confirming that at one point the Universe had been in thermal equilibrium.

Whether inflation is the proper mechanism to generate this extreme expansion, the uniformity of the CMB proves that the Universe began as a hot, dense, quantum-scale object which underwent a period of expansion. After this expansion but prior to the formation of the CMB, the Universe was primarily photons, neutrons, protons, and electrons. At this time the density and temperature of the Universe was high enough that the photons and electrons had a large scattering cross section such that the mean free path of the photons was small. As the Universe expanded it also cooled and when its temperature was $\sim \frac{1}{4} \mathrm{eV}$ [5], protons and electrons combined to form neutral hydrogen (this is known as 'recombination'). The scattering cross section of photons and electrons dropped, and the photons were able to freely stream. This moment of recombination occurred $\sim 400,000$ years after the Big Bang and the relic light from this time constitutes the CMB. As the Universe continued to expand, the wavelength of this light was stretched such that today it is at microwave wavelengths.

\subsection{Anisotropies in the CMB}

There are temperature deviations in the $\mathrm{CMB}$ of $\sim$ one part in the $10^{5}$ (Figure 1). These anisotropies arise from quantum mechanical fluctuations present in the very early Universe which 
were expanded to large scales as the Universe expanded. The fluctuations became potential wells which created large scale structure: the clusters, super-clusters, filaments, and voids observed by astronomers.

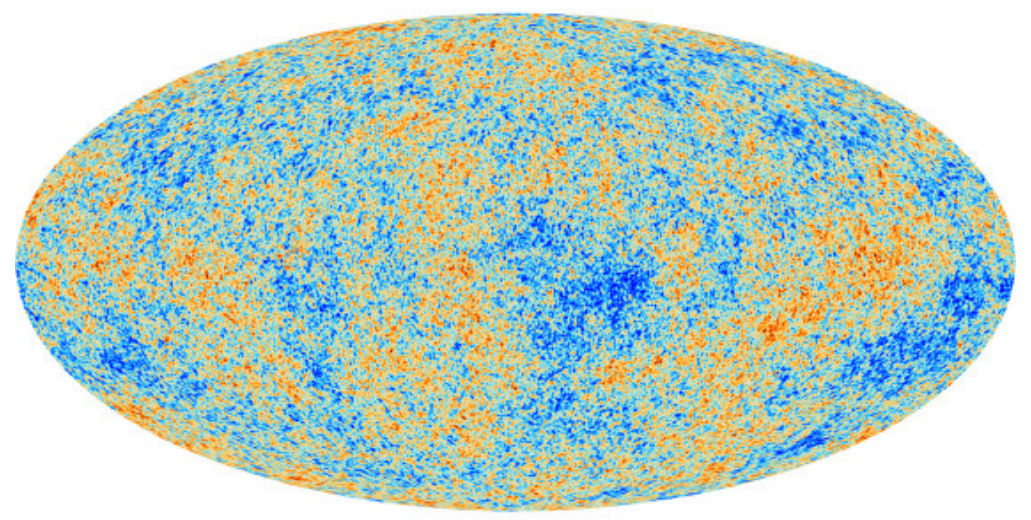

Figure 1: Planck map of the CMB anisotropies, all frequencies with foregrounds removed, credit ESA and the Planck Collaboration

The temperature fluctuations are encoded in the CMB thus: prior to recombination, photons and electrons were tightly coupled in what is typically termed a photon-baryon fluid. As matter fell into the potential wells formed by the early Universe quantum fluctuations, photons were dragged with it. Oscillations in the photon-baryon fluid were created at these potential wells between the competing forces of gravitation and outwards photon pressure. At the moment of recombination when the photons are free to propagate, most temperature fluctuations from most oscillatory phases averaged away except for photons propagating from a peak in the oscillatory phase. A power spectrum of CMB maps (like a two-dimensional spatial Fourier transform which will have peaks at angular distances $\Delta \theta \sim \frac{180}{\ell}$ with significant statistical power in the maps, where $\ell$ is the multipole in $\mathrm{CMB}$ power spectrum) will show that the largest oscillatory mode, which just had time to compress, corresponds to the largest peak at the largest scale in the power spectrum. A smaller $\Delta \theta$ corresponding to a mode that has both compressed and rarefied will be the second peak, etc. Thus the power spectrum of a CMB map contains information about the photon-baryon fluid at the moment of recombination.

Encoded in this plasma at the time of recombination is a treasure trove of information about the constituents of the early Universe. With a prior on the Hubble constant from galaxy surveys or other measurements, the angular diameter of the first peak encodes the geometry of the universe. The ratio of the heights of the first and second peaks provides a measurement of the relative energy densities of dark matter and baryonic matter at recombination. Smaller scales encode the physics at recombination, including the total energy contribution from relativistic particles, the sum of the neutrino masses, and the neutral hydrogen fraction. Measurements of the tilt of the spectrum from large to small scales could constrain simple models of inflation.

Linear polarization is generated in the CMB when photons Thomson scatter off of free electrons located in a quadrupolar temperature distribution (hotter or colder on opposite sides). These quadrupolar moments are set up by motion in the plasma (as opposed to the temperature anisotropies, 


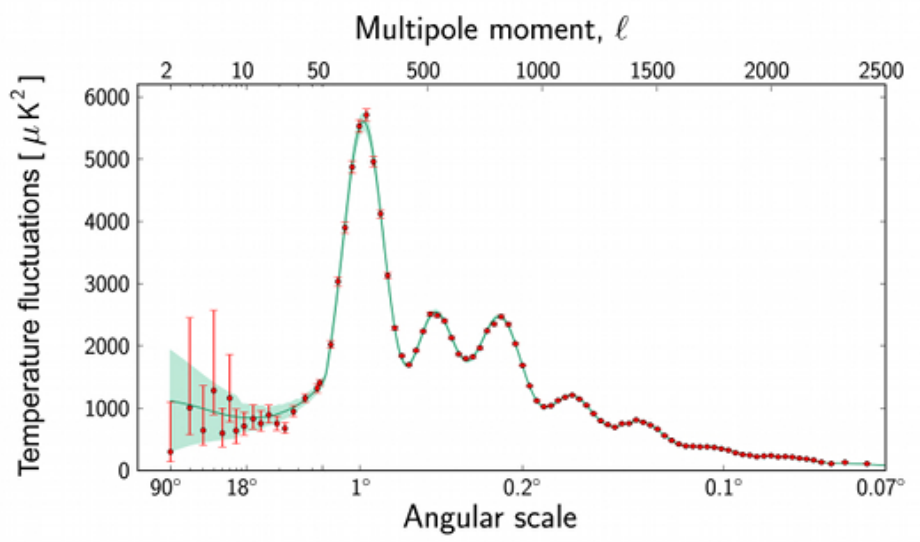

Figure 2: Planck power spectrum of temperature anisotropies, credit ESA and the Planck Collaboration

which are generated when motion in minimal: totally compressed or totally rarefied photon-baryon fluid). There are two methods of generating the appropriate distributions. First, as the plasma accelerates into potential wells, a free falling electron will see red-shifted photons both towards and away from the center of the potential well, since in general the plasma is moving faster the nearer it is to the potential well center. As with the temperature anisotropies, we measure the signal when it is maximal, in this case in the middle of the fall where its velocity is highest. The second way to generate a quadrupolar temperature pattern is with inflationary gravitational waves: as a wave propagates it alternately squeezes and stretches space in the directions perpendicular to its motion. This forms a pattern of red- and blue-shifted light according to an electron at rest in the center that is quadrupolar (and higher order poles at less significance) in nature.

\section{Current Measurements and Cosmological Constraints}

\subsection{Temperature Anisotropy Power Spectrum}

Measurements of the CMB have a long history beginning with the discovery of the background radiation by Penzias and Wilson in 1964 [17]. Many experiments contributed to our understanding of the temperature anisotropy spectrum at many different scales, but for brevity I will focus on recent results. CMB experiments are typically either small-dish telescopes with resolution appropriate for the larger CMB scales $\left(1^{\circ}-15^{\prime}\right)$ and large-dish telescopes which target small scales $\left(\sim 1^{\prime}-10^{\prime}\right)$. The temperature anisotropy spectrum was definitively measured by the Planck collaboration [19], confirming measurements from a combination of large-scale measurements from the WMAP satellite [2] or small-scale measurements from large-dish, high-resolution experiments like the Atacama Cosmology Telescope (ACT) [24] and the South Pole Telescope (SPT) [9]. The resulting constraints on cosmological parameters are cosmic-variance limited and yield $1 \%$ or better precision on the $\Lambda \mathrm{CDM}$ parameters and certain extensions. The constraints from WMAP and Planck are consistent with each other, although there is some tension particularly in the amplitude of the spectrum, and work is on-going to understand the root of that tension [19]. The Planck map and power spectrum from which the constraints are derived is shown in Figures 1 and 2. 
As the CMB photons traverse the Universe, they can be lensed by large scale structure. Unlike galaxy or cluster lensing, CMB lensing is measured as statistical deviations in the maps. The power spectrum of these deviations encodes the gravitational interactions along the line of sight, and therefore the summed mass distribution over the age of the Universe, with a peak in the lensing at scales appropriate for the size of clusters at $\mathrm{z} \sim 2$, or around $10^{\prime}$. This signature was first detected in 2011 [4] and was used to provide the first CMB-only evidence for dark energy [23]. This result has been confirmed and measured with greater precision [25].

\subsection{Cluster Measurements}

The rate of structure formation is dependent on a variety of cosmological parameters, including the sum of the neutrino masses and the equation of state of dark energy, parametrized by $w^{1}$. The number of clusters at a given redshift $\left(\frac{d N}{d z}\right)$ as a function of mass depends exponentially on the dark energy and dark matter densities, and thus a sensitive measurement of this function will inform our understanding of the evolution of dark energy. This cosmological analysis has been performed previously with X-ray selected clusters which, in combination with other cosmological probes, constrained $w=-0.991 \pm 0.045$ (stat) \pm 0.039 (sys) [26] ( $w=-1$ with no time dependence if it is Einstein's cosmological constant).

The CMB provides a method of creating nearly red-shift independent cluster surveys, and hence a path towards understanding structure formation. As CMB photons travel through the Universe, they can Compton scatter off of the hot $\left(>10^{7} \mathrm{~K}\right)$ electrons in the inter-cluster medium. The resultant distortion of the CMB spectrum is known as the thermal Sunyaev-Zel'dovich effect (tSZ), which is proportional to electron pressure integrated along the line of sight and is nearly redshift independent. In simple hydrostatically supported clusters, this distortion provides a straightforward estimate of the cluster mass. Because X-ray emission also traces the gas, the tSZ signal provides a complementary probe to X-ray observations of clusters. However, the estimates of cluster mass from tSZ flux are discrepant from X-ray estimates for the same clusters [14]. The uncertainty in the scaling relation between the tSZ flux and the cluster mass is the primary limiting factor in extracting cosmology from tSZ cluster samples. As a result, the emphasis has changed recently from merely making surveys of clusters with $\mathrm{CMB}$ experiments to fielding high-resolution $\left(<1^{\prime}\right)$ instruments capable of probing the gas physics in clusters to better understand this discrepancy.

There is an additional signature from the motion of clusters relative to the background CMB. This creates a doppler shift as CMB photons interact with the moving cluster gas creating a signal known as the kinetic SZ effect (kSZ). The kSZ has a different spectral signature than the tSZ and is capable of probing relative motion and hence underlying mass distributions. The kSZ has been measured statistically in CMB maps [7] and has been detected for single clusters as well (e.g.[22]. While the current detections are not yet at high enough signal to noise to probe structure, the kSZ will provide an interesting method to look for the so-called 'missing baryons' in future measurements.

\subsection{Polarization Anisotropy Spectrum}

The polarization is measured as Stokes $\mathrm{Q}$ and $\mathrm{U}$ and the resulting polarization maps are de-

\footnotetext{
${ }^{1} p=w \rho$, where $p$ and $\rho$ are the pressure and density of dark energy
} 


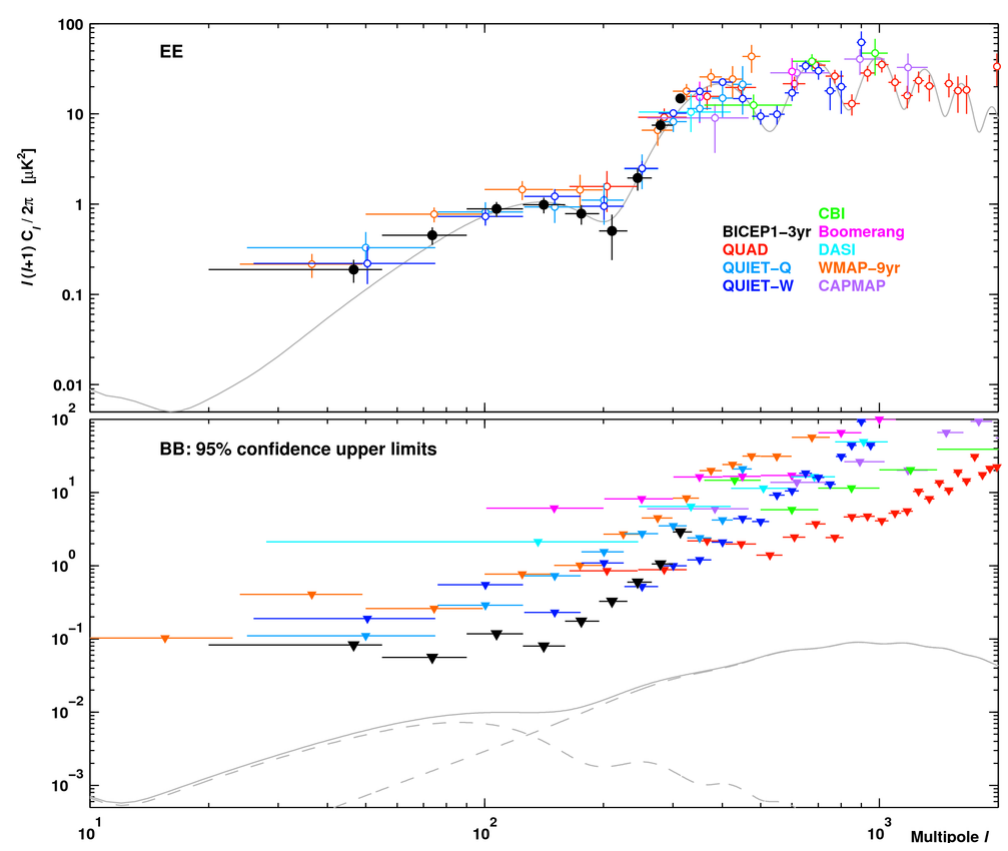

Figure 3: Current E-mode CMB polarization anisotropy spectrum (top) and constraints on B-mode power from inflationary gravity waves (bottom). Figure from [3].

composed into orthogonal E and B modes. E-modes are curl-free and are generated primarily by the motion of plasma in potential wells discussed in Section 2.2. B-modes are divergence free, and are generated from two sources: inflationary gravity waves (also discussed in Section 2.2) which have a peak at angular scales corresponding to $\sim 2^{\circ}$ on the sky, and from lensing of E-modes as the $\mathrm{CMB}$ photons pass through large-scale structure like clusters, which peaks at the same scale as the temperature lensing spectrum. The magnitude of the signal from inflationary gravity waves is unconstrained and such a measurement would not only be evidence for inflation but also yield its energy scale for a certain class of inflationary models. A measurement of B-modes from lensing is constrained by the known magnitudes of the E-mode signal and measurements of large scale structure, and forms a probe of large scale structure and things which impact it (e.g. neutrinos, dark energy).

The E-mode spectrum is $\sim 5$ orders of magnitude smaller than the temperature anisotropies. Because it requires detectors suitable for polarized measurements and far better sensitivity, the first detection of polarization in the CMB was made in 2001 by DASI [11]. The current best measurement of the E-mode spectrum is shown in Figure 3; the E-mode signature is measured to be non-zeros at $15 \sigma$ [3] and is consistent with the current $\Lambda \mathrm{CDM}$ model. Although the temperature anisotropy spectrum is limited by cosmic variance (how many modes can be probed on the sky) by Planck, the E-mode spectrum is still limited by instrument noise, and so constraints from the E-mode spectrum will continue to improve as we advance instrumentation noise and systematics.

A non-zero B-mode signal from lensing was first detected by SPT in 2013 as a cross-correlation between a lensing map and a measurement of structure from Herschel [8]. The first measurement of the B-mode auto-correlation spectrum (measured alone, with no input from other instruments) from lensing was detected by Polarbear also in 2013 [20]. This $4.2 \sigma$ detection is a significant 
achievement for CMB polarization measurements, although there is much more work to be done before it too can be used for precision constraints.

The magnitude of inflationary gravity waves has typically been expressed by the ratio of scalar perturbations to tensor perturbations in the metric, $r$. The current best constraints on this value come from temperature anisotropy measurements: $r<0.11$ [18], and the best constraint from polarization measurements lags behind at $r<0.7$ [3]. We have reached the limits of the constraining power from the temperature anisotropies on $r$, and so the only means we have of ruling out or verifying inflationary scenarios will have to come from polarization measurements. Instruments with improved sensitivity and systematic error removal will be required to make this a reality.

\section{Upcoming and Future Measurements}

There are at least three clear goals for the future of CMB measurements, each at different scales.

- At large scales, we should be able to constrain or measure the signature from inflationary gravity waves in the B-mode polarization spectrum. This is currently unconstrained and a constraint of $r<0.01$ will be an interesting turning point for models of inflation. Constraints from polarization instruments are not yet near this goal, but a variety of instruments are poised to achieve the sensitivity required for this constraint in the next $\sim 5$ years, including Polarbear[10], Keck[16], EBEX[21], and Spider[6].

- At intermediate scales, we will use lensing measurements in both polarization and temperature maps to map cosmic structure. Currently lensing has been detected in both temperature and polarization maps, but far more sensitive detections are required to actually use the data to probe dark energy, neutrino masses, and structure formation. Experiments including ACTPol[15], SPTPol[1], and Polarbear[10] should begin that process.

- Measurements at smaller scales will contribute to understanding structure through tSZ, kSZ, and lensing measurements. If survey regions are appropriately selected, these measurements will join other instruments to begin large-survey field multi-wavelength cosmology. These measurements require high resolution and a good location in the southern hemisphere to overlap with a variety of surveys, of which ACTPol [15] is the only suitable CMB candidate.

Measurements of the CMB join other cosmological probes to support the concordance model of cosmology $\Lambda \mathrm{CDM}$, and have placed the most precise constraints on its parameters. There is still much to be learned, and future instruments have the potential to verify the theory of inflation, constrain the total mass of neutrinos, and join other instruments to begin a new era of multi-wavelength astronomy surveys.

\section{References}

[1] J. E. Austermann, K. A. Aird, J. A. Beall, D. Becker, A. Bender, B. A. Benson, L. E. Bleem, J. Britton, J. E. Carlstrom, C. L. Chang, H. C. Chiang, H.-M. Cho, T. M. Crawford, A. T. Crites, A. Datesman, T. de Haan, M. A. Dobbs, E. M. George, N. W. Halverson, N. Harrington, J. W. 
Henning, G. C. Hilton, G. P. Holder, W. L. Holzapfel, S. Hoover, N. Huang, J. Hubmayr, K. D. Irwin, R. Keisler, J. Kennedy, L. Knox, A. T. Lee, E. Leitch, D. Li, M. Lueker, D. P. Marrone, J. J. McMahon, J. Mehl, S. S. Meyer, T. E. Montroy, T. Natoli, J. P. Nibarger, M. D. Niemack, V. Novosad, S. Padin, C. Pryke, C. L. Reichardt, J. E. Ruhl, B. R. Saliwanchik, J. T. Sayre, K. K. Schaffer, E. Shirokoff, A. A. Stark, K. Story, K. Vanderlinde, J. D. Vieira, G. Wang, R. Williamson, V. Yefremenko, K. W. Yoon, and O. Zahn. SPTpol: an instrument for CMB polarization measurements with the South Pole Telescope. In Society of Photo-Optical Instrumentation Engineers (SPIE) Conference Series, volume 8452 of Society of Photo-Optical Instrumentation Engineers (SPIE) Conference Series, September 2012.

[2] C. L. Bennett, D. Larson, J. L. Weiland, N. Jarosik, G. Hinshaw, N. Odegard, K. M. Smith, R. S. Hill, B. Gold, M. Halpern, E. Komatsu, M. R. Nolta, L. Page, D. N. Spergel, E. Wollack, J. Dunkley, A. Kogut, M. Limon, S. S. Meyer, G. S. Tucker, and E. L. Wright. Nine-year Wilkinson Microwave Anisotropy Probe (WMAP) Observations: Final Maps and Results. Astrophysical Journal Supplement, 208:20, October 2013.

[3] BICEP1 Collaboration. Degree-Scale CMB Polarization Measurements from Three Years of BICEP1 Data. ArXiv e-prints, October 2013.

[4] S. Das et al. Detection of the Power Spectrum of Cosmic Microwave Background Lensing by the Atacama Cosmology Telescope. Physical Review Letters, 107(2):021301, July 2011.

[5] Scott Dodelson. Modern Cosmology. Elsevier Science (USA), 2003.

[6] A. A. Fraisse, P. A. R. Ade, M. Amiri, S. J. Benton, J. J. Bock, J. R. Bond, J. A. Bonetti, S. Bryan, B. Burger, H. C. Chiang, C. N. Clark, C. R. Contaldi, B. P. Crill, G. Davis, O. Doré, M. Farhang, J. P. Filippini, L. M. Fissel, N. N. Gandilo, S. Golwala, J. E. Gudmundsson, M. Hasselfield, G. Hilton, W. Holmes, V. V. Hristov, K. Irwin, W. C. Jones, C. L. Kuo, C. J. MacTavish, P. V. Mason, T. E. Montroy, T. A. Morford, C. B. Netterfield, D. T. O’Dea, A. S. Rahlin, C. Reintsema, J. E. Ruhl, M. C. Runyan, M. A. Schenker, J. A. Shariff, J. D. Soler, A. Trangsrud, C. Tucker, R. S. Tucker, A. D. Turner, and D. Wiebe. SPIDER: probing the early Universe with a suborbital polarimeter. Journal of Cosmology and Astroparticle Physics, 4:47, April 2013.

[7] N. Hand et al. Evidence of Galaxy Cluster Motions with the Kinematic Sunyaev-Zel'dovich Effect. Physical Review Letters, 109(4):041101, July 2012.

[8] D. Hanson, S. Hoover, A. Crites, P. A. R. Ade, K. A. Aird, J. E. Austermann, J. A. Beall, A. N. Bender, B. A. Benson, L. E. Bleem, J. J. Bock, J. E. Carlstrom, C. L. Chang, H. C. Chiang, H.-M. Cho, A. Conley, T. M. Crawford, T. de Haan, M. A. Dobbs, W. Everett, J. Gallicchio, J. Gao, E. M. George, N. W. Halverson, N. Harrington, J. W. Henning, G. C. Hilton, G. P. Holder, W. L. Holzapfel, J. D. Hrubes, N. Huang, J. Hubmayr, K. D. Irwin, R. Keisler, L. Knox, A. T. Lee, E. Leitch, D. Li, C. Liang, D. Luong-Van, G. Marsden, J. J. McMahon, J. Mehl, S. S. Meyer, L. Mocanu, T. E. Montroy, T. Natoli, J. P. Nibarger, V. Novosad, S. Padin, C. Pryke, C. L. Reichardt, J. E. Ruhl, B. R. Saliwanchik, J. T. Sayre, K. K. Schaffer, B. Schulz, G. Smecher, A. A. Stark, K. T. Story, C. Tucker, K. Vanderlinde, J. D. Vieira, M. P. Viero, G. Wang, V. Yefremenko, O. Zahn, and M. Zemcov. Detection of B-Mode Polarization in the Cosmic Microwave Background with Data from the South Pole Telescope. Physical Review Letters, 111(14):141301, October 2013.

[9] Z. Hou, C. L. Reichardt, K. T. Story, B. Follin, R. Keisler, K. A. Aird, B. A. Benson, L. E. Bleem, J. E. Carlstrom, C. L. Chang, H.-M. Cho, T. M. Crawford, A. T. Crites, T. de Haan, R. de Putter, M. A. Dobbs, S. Dodelson, J. Dudley, E. M. George, N. W. Halverson, G. P. Holder, W. L. Holzapfel, S. Hoover, J. D. Hrubes, M. Joy, L. Knox, A. T. Lee, E. M. Leitch, M. Lueker, D. Luong-Van, J. J. McMahon, J. Mehl, S. S. Meyer, M. Millea, J. J. Mohr, T. E. Montroy, S. Padin, T. Plagge, C. Pryke, 
J. E. Ruhl, J. T. Sayre, K. K. Schaffer, L. Shaw, E. Shirokoff, H. G. Spieler, Z. Staniszewski, A. A. Stark, A. van Engelen, K. Vanderlinde, J. D. Vieira, R. Williamson, and O. Zahn. Constraints on Cosmology from the Cosmic Microwave Background Power Spectrum of the $2500 \mathrm{deg}^{2}$ SPT-SZ Survey. Astrophyical Journal, 782:74, February 2014.

[10] Z. D. Kermish, P. Ade, A. Anthony, K. Arnold, D. Barron, D. Boettger, J. Borrill, S. Chapman, Y. Chinone, M. A. Dobbs, J. Errard, G. Fabbian, D. Flanigan, G. Fuller, A. Ghribi, W. Grainger, N. Halverson, M. Hasegawa, K. Hattori, M. Hazumi, W. L. Holzapfel, J. Howard, P. Hyland, A. Jaffe, B. Keating, T. Kisner, A. T. Lee, M. Le Jeune, E. Linder, M. Lungu, F. Matsuda, T. Matsumura, X. Meng, N. J. Miller, H. Morii, S. Moyerman, M. J. Myers, H. Nishino, H. Paar, E. Quealy, C. L. Reichardt, P. L. Richards, C. Ross, A. Shimizu, M. Shimon, C. Shimmin, M. Sholl, P. Siritanasak, H. Spieler, N. Stebor, B. Steinbach, R. Stompor, A. Suzuki, T. Tomaru, C. Tucker, and O. Zahn. The POLARBEAR experiment. In Society of Photo-Optical Instrumentation Engineers (SPIE) Conference Series, volume 8452 of Society of Photo-Optical Instrumentation Engineers (SPIE) Conference Series, September 2012.

[11] E. M. Leitch, J. M. Kovac, N. W. Halverson, J. E. Carlstrom, C. Pryke, and M. W. E. Smith. Degree Angular Scale Interferometer 3 Year Cosmic Microwave Background Polarization Results. Astrophysical Journal, 624:10-20, May 2005.

[12] A. R. Liddle and D. H. Lyth. Cosmological Inflation and Large-Scale Structure. Cambridge University Press, 2000.

[13] J. C. Mather, E. S. Cheng, D. A. Cottingham, R. E. Eplee, Jr., D. J. Fixsen, T. Hewagama, R. B. Isaacman, K. A. Jensen, S. S. Meyer, P. D. Noerdlinger, S. M. Read, L. P. Rosen, R. A. Shafer, E. L. Wright, C. L. Bennett, N. W. Boggess, M. G. Hauser, T. Kelsall, S. H. Moseley, Jr., R. F. Silverberg, G. F. Smoot, R. Weiss, and D. T. Wilkinson. Measurement of the cosmic microwave background spectrum by the COBE FIRAS instrument. Astrophysical Journal, 420:439-444, January 1994.

[14] A. Morandi et al. X-ray and Sunyaev-Zel'dovich scaling relations in galaxy clusters. Monthly Notices of the Royal Astronomical Society, 379:518-534, August 2007.

[15] M. D. Niemack et al. ACTPol: A polarization-sensitive receiver for the Atacama Cosmology Telescope. Proc. SPIE Int. Soc. Opt. Eng., 7741:77411S, 2010.

[16] R. W. Ogburn, P. A. R. Ade, R. W. Aikin, M. Amiri, S. J. Benton, C. A. Bischoff, J. J. Bock, J. A. Bonetti, J. A. Brevik, E. Bullock, B. Burger, G. Davis, C. D. Dowell, L. Duband, J. P. Filippini, S. Fliescher, S. R. Golwala, M. Gordon, M. Halpern, M. Hasselfield, G. Hilton, V. V. Hristov, H. Hui, K. Irwin, J. P. Kaufman, B. G. Keating, S. A. Kernasovskiy, J. M. Kovac, C. L. Kuo, E. M. Leitch, M. Lueker, T. Montroy, C. B. Netterfield, H. T. Nguyen, R. O’Brient, A. Orlando, C. L. Pryke, C. Reintsema, S. Richter, J. E. Ruhl, M. C. Runyan, R. Schwarz, C. D. Sheehy, Z. K. Staniszewski, R. V. Sudiwala, G. P. Teply, K. Thompson, J. E. Tolan, A. D. Turner, A. G. Vieregg, D. V. Wiebe, P. Wilson, and C. L. Wong. BICEP2 and Keck array operational overview and status of observations. In Society of Photo-Optical Instrumentation Engineers (SPIE) Conference Series, volume 8452 of Society of Photo-Optical Instrumentation Engineers (SPIE) Conference Series, September 2012.

[17] A. A. Penzias and R. W. Wilson. A Measurement of Excess Antenna Temperature at 4080 Mc/s. Astrophysical Journal, 142:419-421, July 1965.

[18] Planck Collaboration. Planck 2013 results. XVI. Cosmological parameters. ArXiv e-prints, March 2013.

[19] Planck collaboration, P. A. R. Ade, N. Aghanim, C. Armitage-Caplan, M. Arnaud, M. Ashdown, F. Atrio-Barandela, J. Aumont, C. Baccigalupi, A. J. Banday, and et al. Planck 2013 results. XV. CMB power spectra and likelihood. ArXiv e-prints, March 2013. 
[20] Polarbear Collaboration, P. A. R. Ade, Y. Akiba, A. E. Anthony, K. Arnold, D. Barron, D. Boettger, J. Borrill, S. Chapman, Y. Chinone, M. Dobbs, T. Elleflot, J. Errard, G. Fabbian, C. Feng, D. Flanigan, A. Gilbert, W. Grainger, N. W. Halverson, M. Hasegawa, K. Hattori, M. Hazumi, W. L. Holzapfel, Y. Hori, J. Howard, P. Hyland, Y. Inoue, G. C. Jaehnig, A. Jaffe, B. Keating, Z. Kermish, R. Keskitalo, T. Kisner, M. Le Jeune, A. T. Lee, E. Linder, M. Lungu, F. Matsuda, T. Matsumura, X. Meng, N. J. Miller, H. Morii, S. Moyerman, M. J. Myers, M. Navaroli, H. Nishino, H. Paar, J. Peloton, E. Quealy, G. Rebeiz, C. L. Reichardt, P. L. Richards, C. Ross, I. Schanning, D. E. Schenck, B. Sherwin, A. Shimizu, C. Shimmin, M. Shimon, P. Siritanasak, G. Smecher, H. Spieler, N. Stebor, B. Steinbach, R. Stompor, A. Suzuki, S. Takakura, T. Tomaru, B. Wilson, A. Yadav, and O. Zahn. Gravitational Lensing of Cosmic Microwave Background Polarization. ArXiv e-prints, December 2013.

[21] B. Reichborn-Kjennerud, A. M. Aboobaker, P. Ade, F. Aubin, C. Baccigalupi, C. Bao, J. Borrill, C. Cantalupo, D. Chapman, J. Didier, M. Dobbs, J. Grain, W. Grainger, S. Hanany, S. Hillbrand, J. Hubmayr, A. Jaffe, B. Johnson, T. Jones, T. Kisner, J. Klein, A. Korotkov, S. Leach, A. Lee, L. Levinson, M. Limon, K. MacDermid, T. Matsumura, X. Meng, A. Miller, M. Milligan, E. Pascale, D. Polsgrove, N. Ponthieu, K. Raach, I. Sagiv, G. Smecher, F. Stivoli, R. Stompor, H. Tran, M. Tristram, G. S. Tucker, Y. Vinokurov, A. Yadav, M. Zaldarriaga, and K. Zilic. EBEX: a balloon-borne CMB polarization experiment. In Society of Photo-Optical Instrumentation Engineers (SPIE) Conference Series, volume 7741 of Society of Photo-Optical Instrumentation Engineers (SPIE) Conference Series, July 2010.

[22] J. Sayers, T. Mroczkowski, M. Zemcov, P. M. Korngut, J. Bock, E. Bulbul, N. G. Czakon, E. Egami, S. R. Golwala, P. M. Koch, K.-Y. Lin, A. Mantz, S. M. Molnar, L. Moustakas, E. Pierpaoli, T. D. Rawle, E. D. Reese, M. Rex, J. A. Shitanishi, S. Siegel, and K. Umetsu. A Measurement of the Kinetic Sunyaev-Zel'dovich Signal Toward MACS J0717.5+3745. Astrophysical Journal, 778:52, November 2013.

[23] Blake D. Sherwin et al. Evidence for dark energy from the cosmic microwave background alone using the Atacama Cosmology Telescope lensing measurements. Phys. Rev. Lett., 107:021302, 2011.

[24] J. L. Sievers, R. A. Hlozek, M. R. Nolta, V. Acquaviva, G. E. Addison, P. A. R. Ade, P. Aguirre, M. Amiri, J. W. Appel, L. F. Barrientos, E. S. Battistelli, N. Battaglia, J. R. Bond, B. Brown, B. Burger, E. Calabrese, J. Chervenak, D. Crichton, S. Das, M. J. Devlin, S. R. Dicker, W. Bertrand Doriese, J. Dunkley, R. Dünner, T. Essinger-Hileman, D. Faber, R. P. Fisher, J. W. Fowler, P. Gallardo, M. S. Gordon, M. B. Gralla, A. Hajian, M. Halpern, M. Hasselfield, C. Hernández-Monteagudo, J. C. Hill, G. C. Hilton, M. Hilton, A. D. Hincks, D. Holtz, K. M. Huffenberger, D. H. Hughes, J. P. Hughes, L. Infante, K. D. Irwin, D. R. Jacobson, B. Johnstone, J. Baptiste Juin, M. Kaul, J. Klein, A. Kosowsky, J. M. Lau, M. Limon, Y.-T. Lin, T. Louis, R. H. Lupton, T. A. Marriage, D. Marsden, K. Martocci, P. Mauskopf, M. McLaren, F. Menanteau, K. Moodley, H. Moseley, C. B. Netterfield, M. D. Niemack, L. A. Page, W. A. Page, L. Parker, B. Partridge, R. Plimpton, H. Quintana, E. D. Reese, B. Reid, F. Rojas, N. Sehgal, B. D. Sherwin, B. L. Schmitt, D. N. Spergel, S. T. Staggs, O. Stryzak, D. S. Swetz, E. R. Switzer, R. Thornton, H. Trac, C. Tucker, M. Uehara, K. Visnjic, R. Warne, G. Wilson, E. Wollack, Y. Zhao, and C. Zunckel. The Atacama Cosmology Telescope: cosmological parameters from three seasons of data. Journal of Cosmology and Astroparticle Physics, 10:60, October 2013.

[25] A. Van Englelen et al. A Measurement of Gravitational Lensing of the Microwave Background Using South Pole Telescope Data. The Astrophysical Journal, 756:142, September 2012.

[26] A. Vikhlinin et al. Chandra Cluster Cosmology Project III: Cosmological Parameter Constraints. The Astrophysical Journal, 692:1060-1074, February 2009. 